\title{
Core Clinical Competencies in Anesthesiology: A Case-Based Approach
}

\author{
Christopher J. Gallagher, Michael C. Lewis, Deborah A. Schwengel. \\ Cambridge University Press, Cambridge, UK. ISBN 978-0521144131
}

\author{
Sylvain Boet, MD
}

Published online: 29 July 2011

(C) Canadian Anesthesiologists' Society 2011

The goal of this book is to prepare anesthesiology residents for their specialty examinations in the United States. The Accreditation Council for Graduate Medical Education (ACGME) is the body responsible for the accreditation of postgraduate medical training programs in the United States. The examinations evaluate residents on six core competencies of a competent physician as listed by the ACGME, namely, patient care, medical knowledge, practice-based learning and improvement, interpersonal and communication skills, professionalism, and systems-based practice. The ACGME competencies are similar to the CanMEDS competencies used in Canada and other countries, specifically, medical expert, manager, collaborator, communicator, professional, scholar, and health advocate.

The authors begin their book by providing a didactic and concise description of the six core ACGME competencies in medicine as a whole, followed by a short description within the context of anesthesiology. The book includes 77 clinical cases, and the authors advance either some or all of the six core ACGME competencies related to each specific case. The cases are gathered into six sections (Parts 1 to 6) according to the institutions of the contributing authors, many of whom are anesthesiology residents. The choice of gathering cases into sections according to the affiliation of the authors may be somewhat surprising, since, in my view, it would have been more logical to group the cases according to their main core competencies or even their main clinical focus.

Each case study begins with a catchy title and continues with a case description written in a "humorous" and colloquial style. Whereas this style can be engaging and

S. Boet, MD $(\bowtie)$

The Ottawa Hospital, University of Ottawa, Ottawa, ON, Canada

e-mail: sboet@toh.on.ca entertaining, the reader may often perceive this approach as being unusual at best, especially in this book which aims to advocate clinical core competencies, including professionalism. For example, Case 10 "Flame on!", a case regarding a conscious patient with $100 \%$ burns, starts with "A smell like barbeque fills the entire emergency room. 'Funny,' you think, 'no one told me there was a picnic.' You note that the smell is coming from the trauma bay...". Under the competency "Patient care" and the subcategory "Perform competently all medical and invasive procedures considered essential for the area of practice", the authors write, "As long as I didn't stick the morphine syringe into the mattress by mistake, I was performing competently." This style may appear to be counterproductive to teaching the core competencies that are being discussed.

The hidden curriculum includes learning which is not visible and explicit in an educational program. For example, when medical students participate in an anesthesiology rotation, they learn technical skills, such as drug administration and tracheal intubation, and they also learn about professional relationships with other healthcare providers and the sociology of anesthesiologists, learning points which are often not explicit in their curriculum. I am concerned about the contribution this book, with its sarcastic style, may have on the hidden curriculum, i.e., in shaping the professional identity of residents and potentially contributing to cynical attitudes instead of encouraging compassion and empathy toward the patient.

In each case, the description of the clinical core competencies is clear and well structured with a repetitive format to assist trainees in preparing for their examinations. The amount of redundancy is one drawback which is potentially irritating for the reader; in addition, this 472 page paperback book is rather large $(9.6 \times 7.4 \times 1.1$ inches and 1.9 pounds). The interest in this book lies within 
the description of the clinical core competencies, although, the "Patient care" section is succinct compared with many other medical books which focus specifically on that category of competency.

This book could be an excellent source of clinical cases for educators who teach core clinical competencies to medical students or residents. The cases are designed to target the non- "Patient care" competencies which are addressed less commonly in the majority of medical textbooks.
In conclusion, I believe this book may be of some interest to anesthesiology residents who are preparing for board examinations based on the core competencies in the United States. It could also be an interesting reference book for educators who teach trainees the six ACGME core competencies. However, I am concerned with the style of this book and its possible contribution to the hidden curriculum.

Competing interest None declared. 